\title{
O ensino da terapia a laser de baixa intensidade em Odontologia no Brasil
}

\author{
The teaching of low intensity laser therapy in Dentistry in Brazil
}

\author{
Monalisa da Nóbrega Cesarino Gomes* \\ Marayza Alves Clementino* \\ Thiara Karine de Araújo* \\ Ana Flávia Granville-Garcia** \\ Maria Helena Chaves Vasconcelos Catão** \\ Daliana Queiroga de Castro Gomes ${ }^{* *}$
}

\section{Resumo}

Introdução: a tendência da Odontologia é a incorporação de métodos menos invasivos com a finalidade de minimizar a dor e o desconforto durante e após intervenções odontológicas. Objetivo: este estudo teve como objetivo traçar o perfil do ensino de terapia a laser de baixa intensidade (TLBI) aplicada à Odontologia nos cursos de graduação das faculdades de Odontologia no Brasil. Além de situar, dentro do território brasileiro, os cursos de habilitação em laser disponíveis ao cirurgião-dentista. Materiais e métodos: a amostra foi composta por 174 Instituições de Ensino Superior (IES), que possuem graduação em Odontologia e por 20 cursos de habilitação em TLBI, ambos cadastrados no Conselho Federal de Odontologia. Para a coleta dos dados, foram criados dois formulários: o primeiro, relativo às perguntas sobre as IES que ofereciam curso de laser em seu currículo, na graduação; e o segundo, apresentando perguntas sobre os cursos de habilitações em $L B I$ e a quantidade de profissionais habilitados ativos. Resultados: do total de 174 faculdades pesquisadas, $22(12,6 \%)$, sendo 9,4\% públicas e 14\% privadas $(p=0,399)$, ofertam o ensino do laser na Odontologia. Em relação aos cirurgiões-dentistas habilitados para uso do TLBI em exercício da profissão, no Brasil, existe 190 profissionais distribuídos pelas cinco regiões do país, com o Sudeste abrangendo 70,52\% do total ( $p=$ 0,007 ). Conclusão: percebe-se que uma minoria das IESs do Brasil oferta a disciplina de laser e que o maior número de profissionais habilitados encontra-se nas regiões Sul e Sudeste. Essa inserção de conhecimento traduz maior respeito ao paciente, sendo atendido por profissionais mais capacitados.

Palavras-chave: Lasers. Educação em Odontologia. Currículo.

\section{Introdução}

Apesar dos avanços tecnológicos na Odontologia, o medo e a ansiedade associados ao tratamento dentário ainda são comuns em crianças e adultos, constituindo-se em significativa barreira para a atenção odontológica. Essas reações podem reduzir a cooperação do paciente ao tratamento, infuenciar negativamente na adaptação, aumentar a dificuldade dos procedimentos terapêuticos, ampliar a percepção da dor e, ainda, gerar maior tensão ao profissional ${ }^{1}$.

O cirurgião-dentista deve buscar técnicas que ofereçam maior conforto ao paciente, tentando transformar a Odontologia curativa, dolorosa e traumática em uma que utiliza métodos preventivos, aliada a equipamentos avançados e materiais modernos que proporcionam tratamentos rápidos, conservadores e mais confortáveis para a paciente ${ }^{1}$. A luz laser, com seus respectivos comprimentos de onda, é estudada em várias áreas da Odontologia, como na dentística, em preparos cavitários, condicionamento de esmalte, entre outros ${ }^{2,3}$.

Laser é um acrônimo da língua inglesa formado por light amplification by stimulated emission of radiation (amplificação de luz por emissão estimulada de radiação), que expressa exatamente o modo como a luz é produzida. Consiste em uma 
radiação eletromagnética com características próprias que possui um único comprimento de onda que se propaga coerentemente no espaço e no tempo, de forma colimada e unidirecional, diferenciando-se de uma luz comum ${ }^{4}$.

A tendência da Odontologia é a incorporação de métodos menos invasivos, com a finalidade de minimizar a dor e o desconforto durante e após as intervenções odontológicas. Por isso, acredita-se que a terapia a laser de baixa intensidade (TLBI) seja uma excelente opção de tratamento, na medida em que apresenta efeitos benéficos para os tecidos irradiados, como ativação da microcirculação, produção de novos capilares, efeitos anti-inflamatórios e analgésicos, além de estímulo ao crescimento e à regeneração celular 5 .

O aumento substancial do interesse pela TLBI tem sido notado em círculos científicos, devido ao significativo número de resultados satisfatórios com o tratamento. Porém, a consagração do laser como terapia exige um conhecimento da energia aplicada, uma investigação dos efeitos que produz no organismo e a aplicação de um protocolo correto ${ }^{2}$.

O aproveitamento desse recurso de forma segura pelo cirurgião-dentista está condicionado a um treinamento prévio, pautado no embasamento teórico necessário ao entendimento de tal ciência complementar.

De acordo com as Diretrizes Curriculares Nacionais do curso de graduação em Odontologia aprovadas pela Resolução CNE/CES3/2002 de 19/02/2002, o cirurgião-dentista deverá ter formação generalista, humanista, crítica e reflexiva, para atuar em todos os níveis de atenção à saúde, com base no rigor técnico e científico, além de acompanhar e incorporar inovações tecnológicas (informática, novos materiais, biotecnologia) no exercício da profissão ${ }^{6}$.

Com base no exposto, afirma-se que o conhecimento necessário ao uso seguro e eficaz da TLBI seria uma responsabilidade dos cursos de graduação em Odontologia. No entanto, a realidade é outra; os cirurgiões-dentistas interessados na utilização do laser como terapia complementar em seus consultórios buscam auxílio em cursos de habilitação fornecidos por centros de especialização ${ }^{7}$.

Este estudo teve como objetivo analisar o ensino da TLBI aplicada à Odontologia nos cursos de graduação das Faculdades de Odontologia no Brasil. Além de situar, dentro do território brasileiro, os cursos de habilitação em laser disponíveis ao cirurgião-dentista.

\section{Métodos}

Trata-se de um estudo descritivo, baseado em consultas de dados secundários disponíveis no site do Conselho Federal de Odontologia (CFO), na seção "serviços e consultas", em 2012.

O universo foi composto por todas as Instituições de Ensino Superior (IESs) que oferecem os cursos de graduação em Odontologia cadastrados no CFO, sendo 199 no total. Destes, $174(87,4 \%)$ corresponderam à amostra. E todos os cursos de habilitação de laserterapia, 20 no total, também reconhecidos pelo CFO.

$\mathrm{O}$ site do CFO fornece os nomes, os sites e os e-mails das IESs que ofertam o curso de Odontologia. De posse desses dados, os pesquisadores localizaram, nos sites das IESs, as matrizes curriculares, verificando a presença ou ausência da disciplina de TLBI ou terapias complementares. Quando, no site, não constava a matriz curricular, era enviado um e-mail para a IES questionando sobre a presença ou ausência da disciplina, carga horária, se era obrigatória ou optativa e em que período ela era ofertada.

Para a coleta dos dados, foram criados dois formulários: o primeiro, relativo a informações sobre as IESs que ofereciam o curso de laser em seu currículo na graduação, continha perguntas sobre o nome e tipo de IES (Estadual, Federal, Particular ou Privada), o tipo de disciplina (obrigatória ou optativa), período e carga horária. O segundo formulário apresentava perguntas sobre os cursos de habilitações em TLBI oferecidos em cada estado e a quantidade de profissionais habilitados ativos.

Todos os resultados foram tabulados e avaliados usando o Statistical Package for Social Science ${ }^{\circledR}$ (SPSS for Windows, version 20.0). Os testes estatísticos utilizados para avaliar a associação entre as variáveis foram o qui-quadrado de Pearson e Kruskal-Wallis (nível de significância de 5\%).

\section{Resultados}

Participaram do estudo 199 faculdades, das quais, 25 não responderam aos questionamentos, nem através da página eletrônica da universidade, nem via e-mail, obtendo-se, assim, apenas $174(87,4 \%)$ como amostra final. Foram consideradas, para análise estatística, todas as respostas apresentadas.

Na Tabela 1, pode-se observar a distribuição das ofertas de disciplina de TLBI nas faculdades de Odontologia do Brasil. Do total de 174 faculdades pesquisadas, $22(12,6 \%)$ ofertam o ensino da TLBI na Odontologia em seus currículos. Comparando-se em relação ao tipo de IES, apenas $9,4 \%$ e $14 \%$ das universidades públicas e privadas $(\mathrm{p}=0,399)$, respectivamente, ofertam essa disciplina, tendo uma maior concentração nas regiões Nordeste $(23,20 \%)$ e Sul $(20 \%)$ do país $(\mathrm{p}=0,059)$. 
Tabela 1 - Distribuição de acordo com a oferta da disciplina de laserterapia segundo a região e o tipo de universidade

\begin{tabular}{l|r|r|r}
\hline \multirow{2}{*}{\multicolumn{1}{c|}{ Variável }} & \multicolumn{2}{|c}{ Disciplina de laserterapia } \\
\cline { 2 - 4 } & \multicolumn{1}{c|}{$\begin{array}{c}\text { Sim } \\
\mathrm{n}(\%)\end{array}$} & \multicolumn{1}{c}{$\begin{array}{c}\text { Não } \\
\mathrm{n}(\%)\end{array}$} & $\mathrm{p}^{(1)}$ \\
\hline $\begin{array}{l}\text { Tipo de Universidade } \\
\text { Pública }\end{array}$ & $5(9,4 \%)$ & $48(90,6 \%)$ & 0,399 \\
Privada & $17(14 \%)$ & $104(86 \%)$ & \\
Região & $8(23,5 \%)$ & $26(76,5 \%)$ & \\
Norte & $1(7,1 \%)$ & $13(92,9 \%)$ & 0,059 \\
Nordeste & $6(7,6 \%)$ & $73(92,4 \%)$ & \\
Centro-oeste & $8(20 \%)$ & $28(80 \%)$ & \\
Sudeste & $22(12,6 \%)$ & $152(87,4 \%)$ & \\
Sul & & & \\
Total & & & \\
\hline
\end{tabular}

(1): Através do teste qui-quadrado de Pearson.

* Valores ausentes, iguais a zero.

Segundo a oferta dessa disciplina no currículo, de acordo com as informações coletadas, 77,27\% eram do tipo optativa/eletiva e apenas $22,73 \%$, do tipo obrigatória (Figura 1). Essa disciplina é ministrada entre o segundo e o nono período, sendo o sétimo o mais citado $(30,8 \%)$, com uma média de carga horária de 52,64 horas.

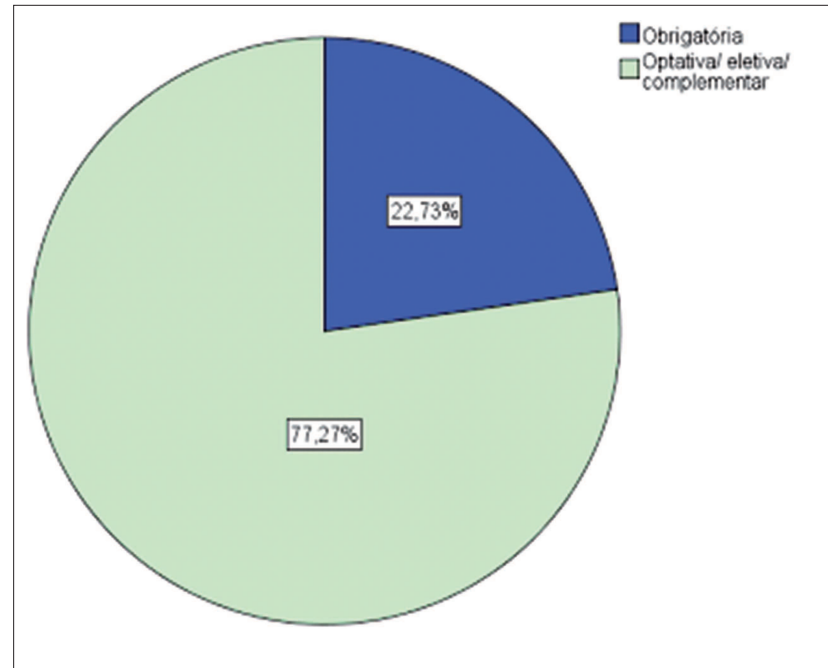

Figura 1 - Distribuição da disciplina de laserterapia de acordo com o tipo

Em todo o país, existem 190 profissionais habilitados para o uso do laser em exercício da profissão, estando distribuídos pelas cinco regiões do país, com o Sudeste abrangendo $70,52 \%$ desse total $(\mathrm{p}=0,007)$. O Sudeste concentra $45 \%$ desses cursos e, em igual número, a região Sul $(\mathrm{p}=0,309)$. Nas regiões Nordeste e Centro-Oeste, não foi encontrado nenhum curso, apesar de conter $9,47 \%$ e $5,26 \%$ do total de profissionais habilitados, respectivamente (Tabela 2).
Tabela 2 - Número de profissionais e cursos de habilitação distribuídos segundo as regiões do país

\begin{tabular}{l|c|c|c|c}
\hline \multirow{2}{*}{ Variável } & \multicolumn{2}{|c|}{ Número de profissionais } & \multicolumn{2}{c}{ Número de cursos } \\
\cline { 2 - 5 } & $\mathrm{n}(\%)$ & $p^{(1)}$ & $\mathrm{n}(\%)$ & $p^{(1)}$ \\
\hline Norte & $2(1,05 \%)$ & & $2(10 \%)$ & \\
Nordeste & $18(9,47 \%)$ & & $*$ & \\
Centro-oeste & $10(5,26 \%)$ & 0,007 & $*$ & 0,309 \\
Sudeste & $134(70,52 \%)$ & & $9(45 \%)$ & \\
Sul & $26(13,68 \%)$ & & $9(45 \%)$ & \\
Total & $190(100 \%)$ & & $20(100 \%)$ & \\
\hline
\end{tabular}

(1): Através do teste Kruskal-Wallis.

* Valores ausentes, iguais a zero.

Do total de 26 estados, e um Distrito Federal, apenas 7 desses apresentaram algum curso de habilitação em TLBI, com uma concentração maior nos estados de São Paulo ( $n=7)$, do Paraná $(n=5)$ e do Rio Grande do Sul (n=4) (Figura 2).

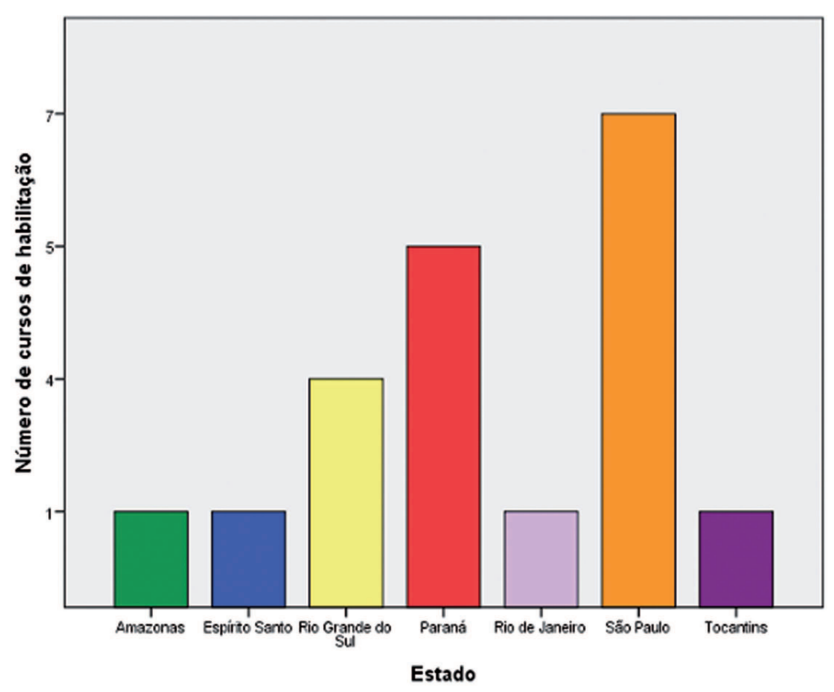

Figura 2 - Distribuição do número de cursos de habilitação de laserterapia de acordo com o estado

\section{Discussão}

O curso de Geraduação em Odontologia tem como perfil do formando egresso/profissional uma formação generalista, humanista, crítica e reflexiva, para atuar em todos os níveis de atenção à saúde, com base no rigor técnico e científico. Esse profissional deve ser capacitado ao exercício de atividades referentes à saúde bucal da população; pautado em princípios éticos, legais e na compreensão da realidade social, cultural e econômica do seu meio e dirigindo sua atuação para a transformação da realidade em benefício da sociedade ${ }^{8}$. Por isso, é nítida a mudança que vem ocorrendo no ensino superior no Brasil, onde a rigidez dos currículos mínimos deixou de existir, dando espaço aos currículos flexíveis, ajustados à realidade. 
Entre as matérias novas propostas pelo projeto de "Diretrizes Curriculares" e pelo "Instrumento de verificação das condições de oferta dos cursos de graduação em Odontologia", estão implante, terceira idade, clínica do bebê, pacientes especiais, bioética, biossegurança, informática, metodologia científica, português e língua estrangeira (instrumentais). Também, estética, implante, biomateriais, laser e Odontologia ocupacional estão entre os temas discutidos pela Comissão de Normatização de Novos Procedimentos e Biomateriais em Odontologia ${ }^{9}$. Portanto, a disciplina de laser, inserida nos cursos de graduação de Odontologia, amplia a visão do aluno e proporciona novos métodos de tratamento.

A introdução de novos conhecimentos no currículo é indiscutível, mas é preciso refletir sobre como fazer essas inserções. O primeiro ímpeto, quase generalizado, é o de criarem-se novas disciplinas. Dessa forma, o currículo ficaria sujeito a constantes acréscimos de disciplinas e, até, de semestres letivos, como também a modismos, à vontade de professores ou, mesmo, a estratégias de marketing de cursos ou faculdades ${ }^{9}$. Por isso, a implantação apenas em $12,6 \%$ da disciplina de laser nas IES do país, sendo um total de $22,7 \%$ dessa disciplina do tipo obrigatória.

Em relação às regiões, teve-se uma concentraçaõ de IESs que contemplem as terapias complementares/laserterapia no Nordeste $(23,20 \%)$ e no Sul (20\%) do país, duas regiões com diferentes economias e culturas. A IES, como instituição social deve, ser colocada no centro de uma discussão diante do conhecimento moderno com sua inexorável velocidade de inovação ${ }^{10}$, uma vez que diferentes modos de atuação do cirurgião-dentista podem trazer benefícios aos pacientes, de acordo com o tipo de tratamento empregado e a capacidade do perfil do profissional que está sendo formado.

Chama a atenção o número de profissionais habilitados na região Sudeste $(70,2 \%)$, cuja porcentagem de cursos de habilitação é a mesma que a da região Sul (45\%). Esse dado demonstra a crescente atuação da TLBI, que, junto à acupuntura, à terapia floral, à hipnose e à homeopatia, foi regulamentada pelo CFO como prática integrativa e complementar à saúde bucal.

Os cursos de habilitação têm como função organizar a prática dessas atividades, impedindo que os profissionais as exerçam sem o conhecimento necessário, produzindo resultados inadequados e trazendo risco aos pacientes, tendo o objetivo de torná-los competentes. Torna-se oportuna, a conceituação de competência, considerando-a como uma capacidade de agir eficazmente em um determinado tipo de situação, com apoio em conhecimentos, mas sem se limitar a eles ${ }^{11}$.
Os únicos estados que apresentaram cursos de habilitação para a TLBI foram Amazonas, Espírito Santo, Rio Grande do Sul, Paraná, Rio de Janeiro, São Paulo e Tocantins.

Como limitações deste estudo, relata-se que algumas IESs não apresentaram atualizações de suas páginas eletrônicas, ou não se teve resposta regular em seus endereços. Dessa forma, produziu-se um total de $12,56 \%$ de dados perdidos.

Ainda há muito que se discutir a respeito das terapias complementares, como a TLBI, mas já é um avanço conseguir regulamentá-las. Hoje, sabe-se que os profissionais habilitados estão mais conscientes da importância do estudo para que melhores resultados sejam obtidos.

\section{Conclusões}

Percebe-se que uma minoria das IESs do Brasil oferta a disciplina de laser/terapias complementares, estando mais concentrada nas universidades públicas da região Nordeste. Já em relação ao número de cursos de habilitação e profissionais habilitados, há uma maior frequência nas regiões Sul e Sudeste. Com isso, evidencia-se que, nas regiões de economia maior, existe já uma consolidação do uso do laser, enquanto nas menos favorecidas economicamente há uma tendência de inserção do laser mais recente nas disciplinas dentro dos currículos.

Portanto, há uma tendência de inserção desse novo conhecimento e a regulamentação de cursos para o uso do laser por cirurgiões-dentistas traduz um maior respeito principalmente ao paciente, que agora é atendido por profissionais mais capacitados a melhorar sua qualidade de vida.

\section{Abstract}

Introduction: the trend in dentistry is the incorporation of less invasive methods with the purpose of minimizing the pain and discomfort during and after dental interventions. Objective: this study aimed to profile the teaching of low intensity laser therapy (LILT) applied to dentistry in undergraduate courses of Dentistry schools in Brazil. It also aimed to situate qualification courses for laser therapy available to dentists within the Brazilian territory. Materials and methods: the sample consisted of 174 Higher Education Institutions (HEIS), which offer graduation programs in Dentistry; and of 20 qualification courses for LILT. All samples were registered in the Federal Council of Dentistry. For data collection we created two forms: the first one concerning questions about the HEls offering laser courses in their graduation curriculum, and the second one presented questions about qualifications courses for $L I L$ and the number of skilled professionals at work. Results: from the total of 174 schools surveyed, 22 (12.6\%) - 9.4\% public and $14 \%$ private ( $p=0.399)$, offer education in laser dentistry. Regarding working dentists qualified for LILT, in Brazil there are 190 trained professionals distri- 
buted among the five regions of the country; the South East region covers $70.52 \%$ of these professionals ( $p=$ 0.007). Conclusion: it is noticed that a minority of the HEIs in Brazil offer a subject for laser education, and that most qualified professionals are in the south and southeast regions. This knowledge input translates into more respect for patients as more qualified professionals may assist them.

Keywords: Laser. Dental education. Curriculum.

\section{Referências}

1. Castilho Filho T. Avaliação da ação da radiação laser em baixa intensidade no processo de ósseo-integração de implantes de titânio inseridos em tíbia de coelhos [Dissertação de Mestrado]. São Paulo: Instituto de pesquisa energéticas nucleares da Faculdade de Odontologia da Universidade de São Paulo; 2003.

2. Cavalcanti TM, Almeida-Barros RQ, Catão MHCV, Feitosa APA, Lins RDAU. Conhecimento das propriedades físicas e da interação do laser com os tecidos biológicos na Odontologia. An Bras Dermatol 2011; 86(5):955-60.

3. Moriyama LT. Ablação de resinas compostas com laser de Er: YAG sob diferentes fluxos de água [Dissertação de Mestrado]. São Paulo: Instituto de Física de São Carlos da Universidade de São Paulo; 2006.

4. Domingues MC, Góes MPS, Botelho KVG, Couto GBL. CVDENTUS ${ }^{\circledR}$ System and child's behavior. Odontologia. Clín Científ 2009; 8(3):251-5.

5. Henriques ACG, Maia AMA, Cimões R, Castro JFL. A laserterapia na Odontologia: propriedades, indicações e aspectos atuais. Odontologia Clín Científ 2008; (7):197-200.

6. Brasil. Ministério da Educação e Cultura. Conselho Nacional de Educação. Resolução CNE/CES 3/2002. Diário Oficial da União, Brasília, 04 mar 2002 [citado 2005 Ago 10]. Disponível em URL: http://www.mec.gov.br/sesu/ftp/resolucao/03020 odontologia.doc.

7. Bicudo LL. Laserterapia exige conhecimento. Informativo do Conselho Regional de Odontologia 2010:4.

8. Conselho Nacional de Educação. Resolução CNE/CES 3/2002. Diário Oficial União 2002; Seção 01(10):1-5.

9. Ribeiro ECO. Novos espaços sociais de aprendizagem e difusão do conhecimento. Caderno LCE, NUTES - UFRJ; 1999.

10. Silveira JLGC. Diretrizes Curriculares Nacionais para os cursos de graduação em Odontologia: historicidade, legalidade e legitimidade. Pesq Bras Odontoped Clin Integr 2004; 4(2):151-6.

11. Carvalho ACP. Planejamento do curso de graduação de Odontologia. Revista da ABENO 2004; 4(1):7-13.
Endereço para correspondência:

Daliana Queiroga de Castro Gomes

Rua Presidente Kennedy, 512, Bairro

Tambauzinho

58042-180 João Pessoa-PB

Fone: (83) 3512-2488

E-mail:dqcgomes@hotmail.com

Recebido: 07/01/2013. Aceito: 24/04/2013. 